\title{
CORRIGENDUM
}

\section{Probing superfluids in optical lattices by momentum-resolved Bragg spectroscopy}

Philipp T. Ernst, Sören Götze, Jasper S. Krauser, Karsten Pyka, Dirk-Sören Lühmann, Daniela Pfannkuche \& Klaus Sengstock

Nature Physics doi:10.1038/nphys1476; published online: 29 November 2009; corrected online: 8 December 2009.

In the version of this Article originally published, Fig. $4 \mathrm{~b}$ should have been as shown here. This has been corrected in all versions of this Article.

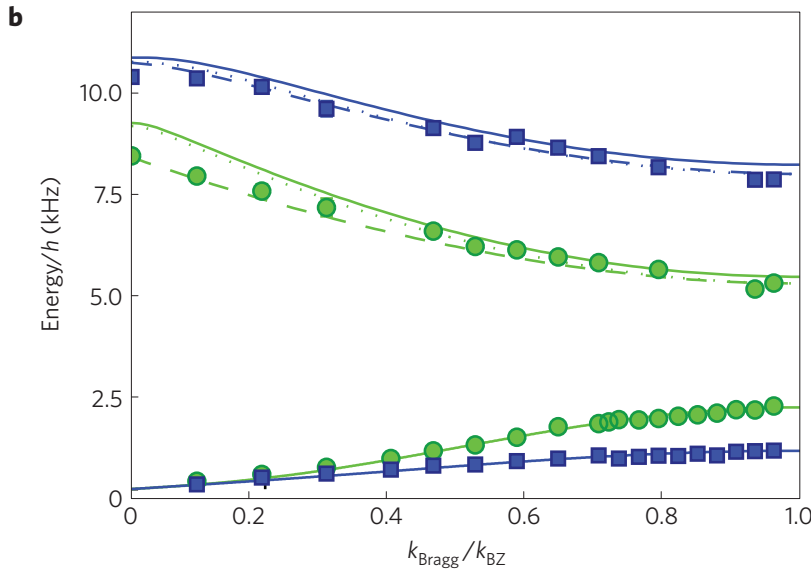

